\title{
Fatty Acid-Induced Injury in Developing Piglet Intestine: Effect of Degree of Saturation and Carbon Chain Length ${ }^{1}$
}

\author{
OTTO R. VELASQUEZ, PATRICK TSO, AND KAREN D. CRISSINGER \\ Departments of Physiology [O.R.V., P.T., K.D.C.] and Pediatrics [K.D.C.], Louisiana State University Medical \\ Center, Shreveport, Louisiana 71130
}

\begin{abstract}
Luminal perfusion with the long-chain fatty acid (LCFA) oleate in concentrations similar to that found in premature infant formula produces a dose- and agedependent mucosal injury in developing intestine. To investigate whether this lipid-induced phenomenon is a function of the degree of saturation and/or chain length of the fatty acid, ${ }^{51} \mathrm{Cr}$-EDTA plasma-to-lumen clearance was measured in jejunum and ileum of 1-d-, 3-d-, 2-wk-, and 1-mo-old piglets after perfusion with 5-mM solutions of different medium-chain saturated fatty acids and saturated and unsaturated LCFA. Mono- and polyunsaturated LCFA produced significant increases in jejunal permeability. In general, this effect was greater in piglets $\leq 2 \mathrm{wk}$ old compared with 1-mo-old animals, but no differences were observed among the unsaturated LCFA within an age group. In contrast, the alterations in mucosal permeability induced by medium-chain fatty acids were overall more attenuated than those induced by LCFA. Our results suggest that developing intestine is vulnerable to the injurious effect of dietary fatty acids and that the lipid-induced changes in mucosal permeability appear to be a function of the fatty acid chain length. The degree of saturation of the fatty acid does not alter its cytotoxic effects. (Pediatr Res 33: 543547, 1993)
\end{abstract}

\section{Abbreviations}

MCFA, medium-chain fatty acid

LCFA, long-chain fatty acid

TG, triglyceride

The adaptation from a high-carbohydrate diet in the fetus to a high-lipid diet in the newborn is a critical event for the normal growth and development of the neonate. Lipids are the most important source of energy in the neonatal period, accounting for 40 to $55 \%$ of the total calories present in human milk and most infant formulas (1).

Digestion and absorption of lipids are less efficient in the newborn, especially the premature infant, compared with the adult. Factors contributing to this impaired fat absorption include a small bile acid pool (2) and inadequate exocrine activity of the pancreas (3). However, recent studies have shown that even preterm infants, especially the breast-fed neonate, are able

Received October 13, 1992; accepted February 1, 1993.

Correspondence: Karen D. Crissinger, M.D., Ph.D., Department of Pediatrics, LSU Medical Center, P.O. Box 33932, Shreveport, LA 71130.

Supported by Grant DK-43785 from the National Institutes of Health and Ross Laboratories.

${ }^{1}$ Presented in part at the meeting of the Southern Society for Pediatric Research, January 1992, New Orleans, LA. to digest efficiently more than $80 \%$ of the total dietary fat as a result of the unique functions of gastric lipase and the bile saltstimulated lipase present in human milk $(4,5)$. Thus, a high concentration of FFA should be present in the intestinal lumen postprandially.

Despite the important nutritional value of dietary fatty acids, these compounds have been implicated in the pathophysiology of intestinal mucosal injury in developing $(6,7)$ and adult animals $(8,9)$. We have shown that perfusion of developing piglet intestine with oleic acid, a long-chain dietary fatty acid, produces dose-dependent increases in mucosal permeability as quantitated by the plasma-to-lumen clearance of ${ }^{51} \mathrm{Cr}$-labeled EDTA. These changes in mucosal permeability are associated with disruption of the epithelial lining of the villus tip and mucosal cell injury observed with light and electron microscopy (7). Interestingly, it appears that developirig piglet intestine becomes more resistant to oleic acid-induced injury as a function of postnatal age (7). Although the injury induced by dietary fatty acids seems to be reversible, this phenomenon is of greater importance in developing intestine, where other injurious events (ischemic, infectious) can be superimposed and might lead to irreversible damage to the intestinal mucosa.

The processes involved in the pathogenesis of fatty acidinduced injury are not known. In this study, we investigated the effect of different dietary fatty acids on intestinal mucosal injury in developing piglet intestine. The question addressed was whether the lipid-induced injury is dependent on the degree of saturation or the carbon chain length of the fatty acid. The results of these experiments should provide insight into the interaction between lipids and mucosal injury in immature intestine.

\section{MATERIALS AND METHODS}

Animal preparation. Hampshire/Yorkshire piglets of either sex were randomly selected among groups of 1-d-old (1-7 h of age; $1.2 \pm 0.17 \mathrm{~kg} ; n=14)$, 3-d-old $(1.88 \pm 0.07 \mathrm{~kg} ; n=10), 2$-wkold $(4.7 \pm 0.18 \mathrm{~kg} ; n=14)$, and 1-mo-old $(6.1 \pm 0.77 \mathrm{~kg} ; n=$ 12) animals. All animals were either never nursed (1-d-old), fasted for $18 \mathrm{~h}$ (3-d-old), or fasted for $24 \mathrm{~h}$ (2-wk and 1-mo-old). After intramuscular injection of ketamine hydrochloride $(20 \mathrm{mg} /$ $\mathrm{kg})$ and xylazine $(2 \mathrm{mg} / \mathrm{kg})$, the animals were anesthetized with pentobarbital sodium $(15 \mathrm{mg} / \mathrm{kg})$ via an ear vein. Maintenance doses of pentobarbital $(5 \mathrm{mg} / \mathrm{kg})$ were given as necessary during the experiment. This study was approved by the Louisiana State University Medical Center Animal Resources Committee.

Immediately after anesthetic induction, the animals were artificially ventilated via a tracheostomy with an intermediate ventilator (Harvard Apparatus, South Natick, MA) at a tidal volume and respiratory rate to maintain normal arterial blood gases and $\mathrm{pH}(\mathrm{pH} /$ blood gas analyzer; Instrumentation Laboratory, Lexington, MA). Polyethylene cannulas were inserted into the right external jugular vein for administration of pentobarbital 
and fluid for hydration and into the left carotid artery to monitor systemic arterial pressure (physiologic recorder, Grass Instrument Co., Quincy, MA) and to withdraw blood samples. Body temperature was maintained at $\sim 37^{\circ} \mathrm{C}$ with a heating pad and an infrared heating lamp.

The abdomen was opened through a midline incision and the renal vessels were ligated to prevent the excretion of chromium 51-labeled EDTA. Loops of jejunum and distal ileum (four loops per intestinal segment), $10-15 \mathrm{~cm}$ in length, were isolated and cannulated at both proximal and distal ends with polyethylene tubing, after which the intestinal and abdominal contents were irrigated with normal saline and covered with plastic wrap to prevent evaporative water loss.

Preparation of fatty acid solutions. Solutions of $5 \mathrm{mM}$ FFA in normal saline were prepared. A concentration of $5 \mathrm{mM}$ was chosen because theoretically this concentration is well within a normal physiologic range after formula feeding in newborn intestine. For instance, a widely used premature infant formula contains $3.6 \%$ fat mostly in TG form with an average molecular weight of 685 . Thus, for each $10 \mathrm{~mL}$ of formula ingested, about $0.35 \mathrm{~g}$ of $\mathrm{TG}$ are available for digestion. That is $0.5 \mathrm{mmol}$ of TG. Iverson et al. (10) have shown that intragastric lipolysis accounts for the digestion of as much as $60 \%$. of dietary fat. Assuming that at least $60 \%$ of TG are digested and that each TG molecule liberates two molecules of fatty acids, $0.6 \mathrm{mmol}$ of fatty acids are liberated after gastric digestion, which is equivalent to a concentration of $60 \mathrm{mM}$.

The different fatty acids used were obtained from Sigma Chemical Co. (St. Louis, MO) at their highest purity available and included linolenic acid (C18:3, n-3), linoleic acid (C18:2, n$6)$, oleic acid (C18:1, n-9), stearic acid (C18:0), palmitoleic acid (C16:1, n-7), palmitic acid (C16:0), myristoleic acid (C14:1, n5), myristic acid (C14:0), lauric acid (C12:0), capric acid (C10:0), and caprylic acid (C8:0). The solutions were solubilized with 10 $\mathrm{mM}$ taurocholic acid (sodium salt, 98\%, Sigma), and the $\mathrm{pH}$ and osmolarity were adjusted to $\sim 7.4$ and $\sim 300 \mathrm{mosmol} / \mathrm{L}$, respectively.

Experimental protocol. Piglets were divided into two experimental groups. In the first group ( $n=5$ /age group), solutions of LCFA of different degrees of saturation (C18:3, C18:2, C18:1, and $\mathrm{C} 18: 0)$ were tested. In the second group ( $n=5-7 /$ age group), fatty acids of different chain length (C16:0, C12:0, C10:0, and $\mathrm{C} 8: 0)$ were used. An additional group of 1 -d-old piglets $(n=4)$ was used to compare the effects of two saturated fatty acids (C14:0 and C16:0), which are more soluble in water than stearic acid, versus their monounsaturated analogs (C14:1 and $\mathrm{C} 16: 1)$.

After surgery, the cannulated loops were perfused with warmed normal saline via a Minipuls 2 (Gilson, Middleton, WI) peristaltic pump at a rate of $1 \mathrm{~mL} / \mathrm{min}$ to achieve a constant flow. ${ }^{51} \mathrm{Cr}-$ EDTA (New England Nuclear, Boston, MA) was then injected i.v. such that plasma cpm were at least $25000 / \mathrm{mL}(100-150$ $\mu \mathrm{Ci} / \mathrm{kg}$ ). Ten min were allowed for tissue equilibration of the labeled EDTA. After the equilibration period, the four loops in each intestinal segment were initially perfused with normal saline during $20 \mathrm{~min}$ (control period). After this control period, the different fatty acid solutions in each experimental group were perfused simultaneously in different loops in both intestinal segments for $20 \mathrm{~min}$ (F.A. period). Subsequently, the perfusate was changed to normal saline for 60 additional min (recovery period). Luminal perfusate from the effluent cannula $(4 \mathrm{~mL})$ was collected at 10 -min intervals along the experiment to monitor ${ }^{51} \mathrm{Cr}$-EDTA clearance.

One $\mathrm{mL}$ of blood (centrifuged to obtain $0.5 \mathrm{~mL}$ of plasma) was obtained every $20 \mathrm{~min}$ to monitor plasma ${ }^{51} \mathrm{Cr}$-EDTA radioactivity. The blood was replaced with an equivalent volume of $6 \%$ Dextran 70 (Sigma). ${ }^{51} \mathrm{Cr}$-EDTA activity in plasma and in 4$\mathrm{mL}$ aliquots of perfusate for each $10 \mathrm{~min}$ was measured in an LKB CompuGamma spectrometer (model 1282; LKB Instruments, Inc., Gaithersburg, MD).

At the end of the experiment, the animal was killed with an overdose of pentobarbital $(60 \mathrm{mg} / \mathrm{kg})$, and each loop of intestine was removed, rinsed with normal saline, and weighed.

Calculation of ${ }^{51} \mathrm{Cr}$-EDTA clearance. The plasma-to-lumen clearance of ${ }^{51} \mathrm{Cr}$-EDTA was calculated as follows:

$$
\text { Clearance }=\frac{\mathrm{cpm}_{\mathrm{p}} \times \mathrm{pr} \times 100}{\mathrm{cpm}_{\mathrm{pl}} \times \mathrm{wt}}
$$

where clearance is given in $\mathrm{mL}$ per min per $100 \mathrm{~g}, \mathrm{cpm}_{\mathrm{p}}$ is $\mathrm{cpm}$ per $\mathrm{mL}$ of perfusate, $\mathrm{pr}$ is the perfusion rate, $\mathrm{cpm}_{\mathrm{pl}}$ is $\mathrm{cpm}$ per $\mathrm{mL}$ of plasma, and wt is weight of the intestinal segment in $\mathrm{g}$.

Data analysis. All values for ${ }^{51} \mathrm{Cr}$-EDTA clearance are expressed as mean \pm SEM. Clearance values were evaluated using a three-factor analysis of variance for repeated measures, followed by Duncan multiple range tests if the analysis of variance revealed differences among age groups, fatty acid solutions, and experimental periods (SAS Institute Inc., Cary, NC). Differences were considered significant at $p<0.05$.

\section{RESULTS}

Effect of degree of saturation. Perfusion of developing intestine with LCFA significantly increased mucosal permeability compared with saline control perfusion. The effect was observed within minutes after the infusion of the lipid solutions and reached a peak in the first few minutes of the recovery period (Fig. 1). This was probably due to a small volume of the fatty acid solution that was left in the system at the end of the F.A. period.

In jejunum, the increase in mucosal permeability in response to perfusion with unsaturated LCFA was similar among age groups in piglets $\leq 2 \mathrm{wk}$ old, whereas this increase in 1 -mo-old animals was significantly less than that of the younger animals (Figs. 2 and 5). The changes in mucosal permeability observed in ileum were overall more attenuated than that observed in the jejunum (data not shown).

Of interest is that perfusion with the saturated fatty acid stearate $(\mathrm{C} 18: 0)$ produced a significantly lower change in mucosal permeability compared with its unsaturated analogs in 1-d-, 3-d-, and 2-wk-old piglets. It is important to point out that stearic acid (C18:0) is extremely insoluble in water under the conditions used to prepare the solutions in this study. To investigate whether there was truly a difference between saturated and unsaturated lipids, jejunum of 1-d-old animals was perfused with saturated [myristic acid (C14:0) and palmitic acid (C16:0)] and monounsaturated [myristoleic acid (C14:1) and palmitoleic acid (C16:1)] fatty acids. These shorter-chain-length fatty acids are more soluble in water than is stearic acid. The increase in mucosal permeability was not different for the saturated fatty acids versus their monounsaturated analogs (Fig. 3). These results suggest

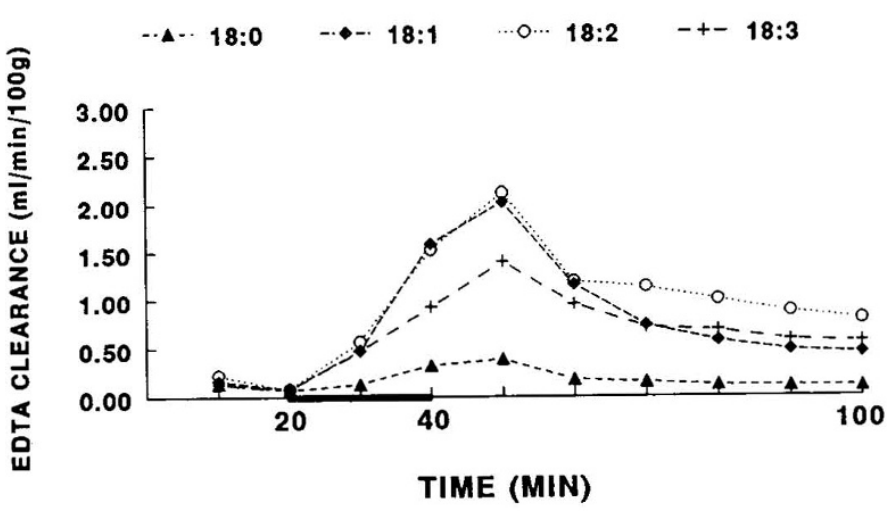

Fig. 1. Effect of perfusion of LCFA solutions $(5 \mathrm{mM})$, with different degrees of saturation, on plasma-to-lumen ${ }^{51} \mathrm{Cr}$-EDTA clearance in 1-dold piglet jejunum. The thick bar on the $x$ axis represents the period at which the different fatty acids were perfused. At all other times, the intestine was perfused with normal saline. 

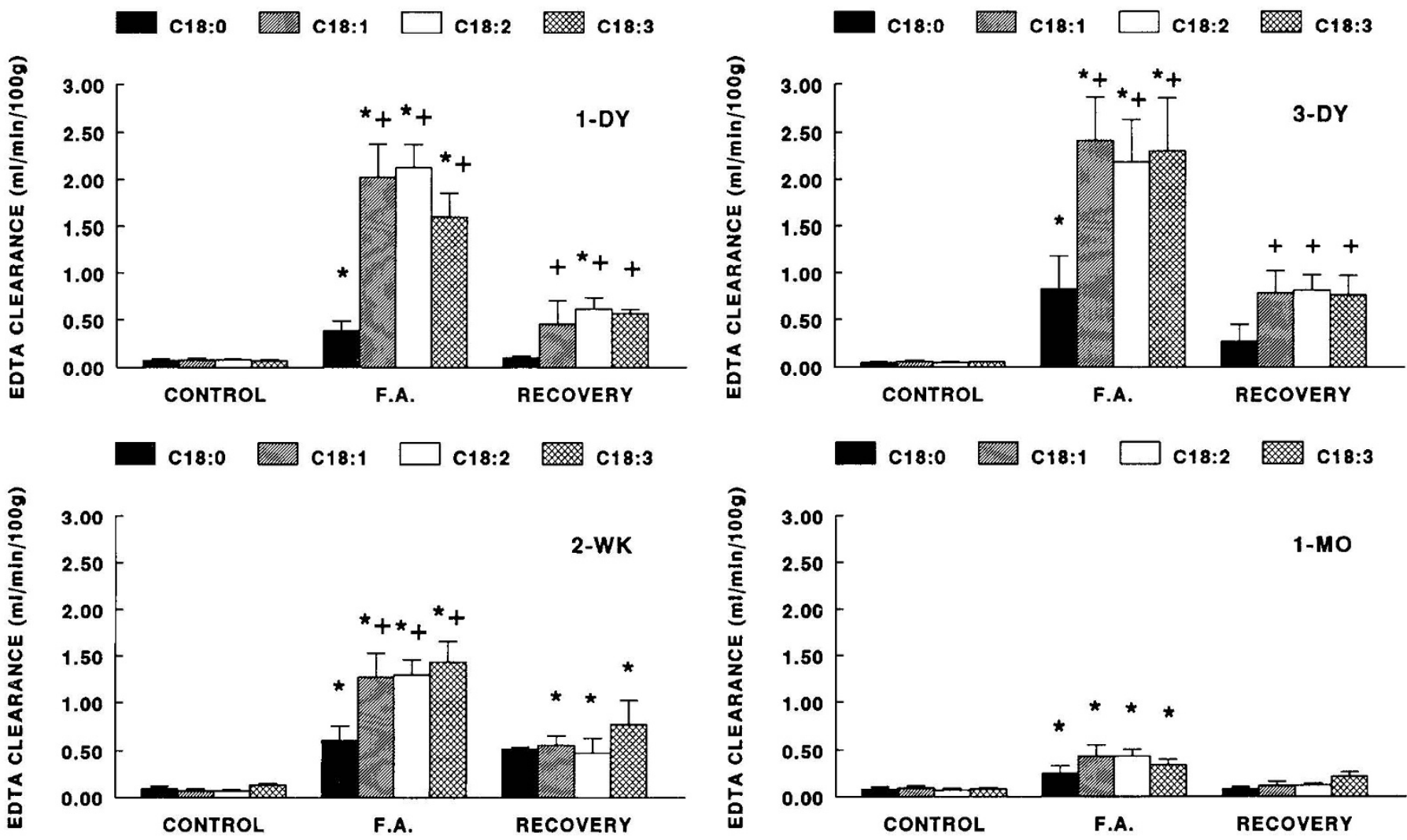

Fig. 2. ${ }^{51} \mathrm{Cr}$-EDTA clearance after perfusion with saturated and unsaturated LCFA in jejunum. Values were obtained after control, F.A. (peak increase at time $50 \mathrm{~min}$ ), and recovery periods in intestine of 1 -d-, 3-d-, 2-wk-, and 1-mo-old piglets. ${ }^{*}, p<0.05$ vs control within a fatty acid solution.,$+ p<0.05$ vs stearic acid (C18:0) within an experimental period.

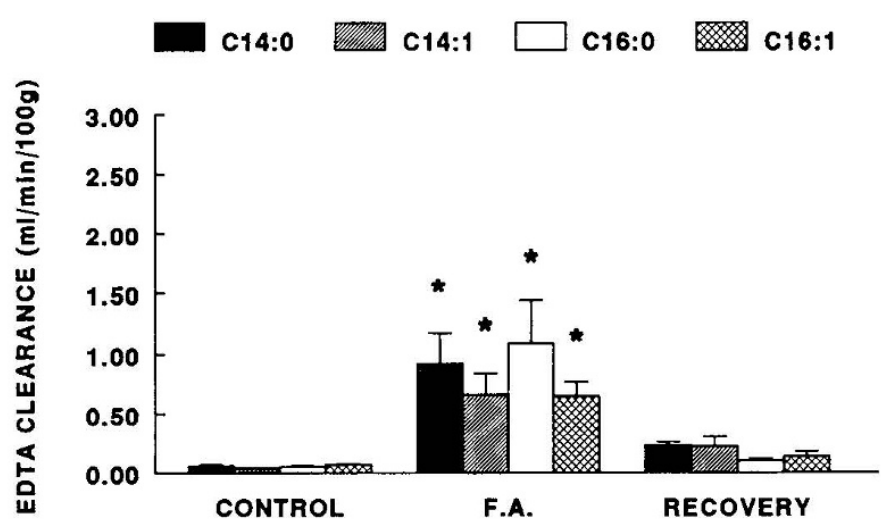

Fig. 3. ${ }^{51} \mathrm{Cr}$-EDTA clearance in jejunum after perfusion with myristic acid $(\mathrm{C} 14: 0)$ and palmitic acid $(\mathrm{C} 16: 0)$ and their monounsaturated analogs myristoleic (C14:1) and palmitoleic acid (C16:1), respectively, in 1d-old piglets. ${ }^{*}, p<0.05$ vs control within a fatty acid solution.

that the marked differences observed between stearic and oleic acid are probably not due to the degree of saturation. Whether stearic acid, however, possesses an intrinsic characteristic that makes it less injurious to the intestinal mucosa remains to be investigated.

Mucosal EDTA clearances returned near baseline values after $1 \mathrm{~h}$ of perfusion with normal saline (recovery period). Although in some age and fatty acid groups mucosal permeability was still significantly higher than control at the end of the saline perfusion, these values were statistically lower compared with those of the F.A. period.

Effect of carbon chain length. In jejunum, perfusion with fatty acids of carbon chain lengths varying from $\mathrm{C} 8$ to $\mathrm{C} 16$ produced significant increases in mucosal permeability compared with the saline control perfusion in piglets $2 \mathrm{wk}$ of age and younger (Figs. 4 and 5). Only lauric acid (C12:0) and palmitic acid (C16:0) significantly increased mucosal injury compared with saline control perfusion in 1-mo-old piglets, and the overall response was more attenuated than the responses in the younger animals.

Among the various fatty acids, the increase in permeability induced by the fatty acid of the smallest chain length [caprylic acid (C8:0)] was significantly lower compared with lauric (C12:0) and palmitic acid $(\mathrm{C} 16: 0)$ in very young piglets $\leq 3 \mathrm{~d}$ old (Fig. 4). This difference could not be attributed to insolubility of the fatty acid because caprylic acid $(\mathrm{C} 8: 0)$ is very soluble in water. In the older groups, no statistically significant differences were observed among the different fatty acids during the F.A. period. Capric acid (C10:0) produced increases in permeability that, although significantly higher than control in most of the age groups, did not differ from the response of caprylic acid (C8:0) at any age. Most of the permeability values returned to near baseline after $1 \mathrm{~h}$ of perfusion with normal saline. In ileum, a more attenuated response was observed compared with that for jejunum (data not shown).

Summary comparison between MCFA and LCFA. Figure 5 depicts the changes in mucosal permeability observed after perfusion of all the fatty acids (except stearate) used in this study in jejunum of 1-d- and 1-mo-old piglets. In addition to the developmental differences already described, a trend can be observed in which the lipid-induced injury generally increases as a function of carbon chain length. In both age groups, the LCFA oleic acid (C18:1) and linoleic acid (C18:2), produced significantly more injury than capric (C10:0) and caprylic acid (C8:0), both MCFA. However, only in 1-d-old piglets were the responses to lauric acid (C12:0), another MCFA, and palmitic acid (C16:0) also significantly higher than caprylic acid (C8:0). In contrast, the effects of LCFA of similar chain length were not altered by different degrees of saturation. 

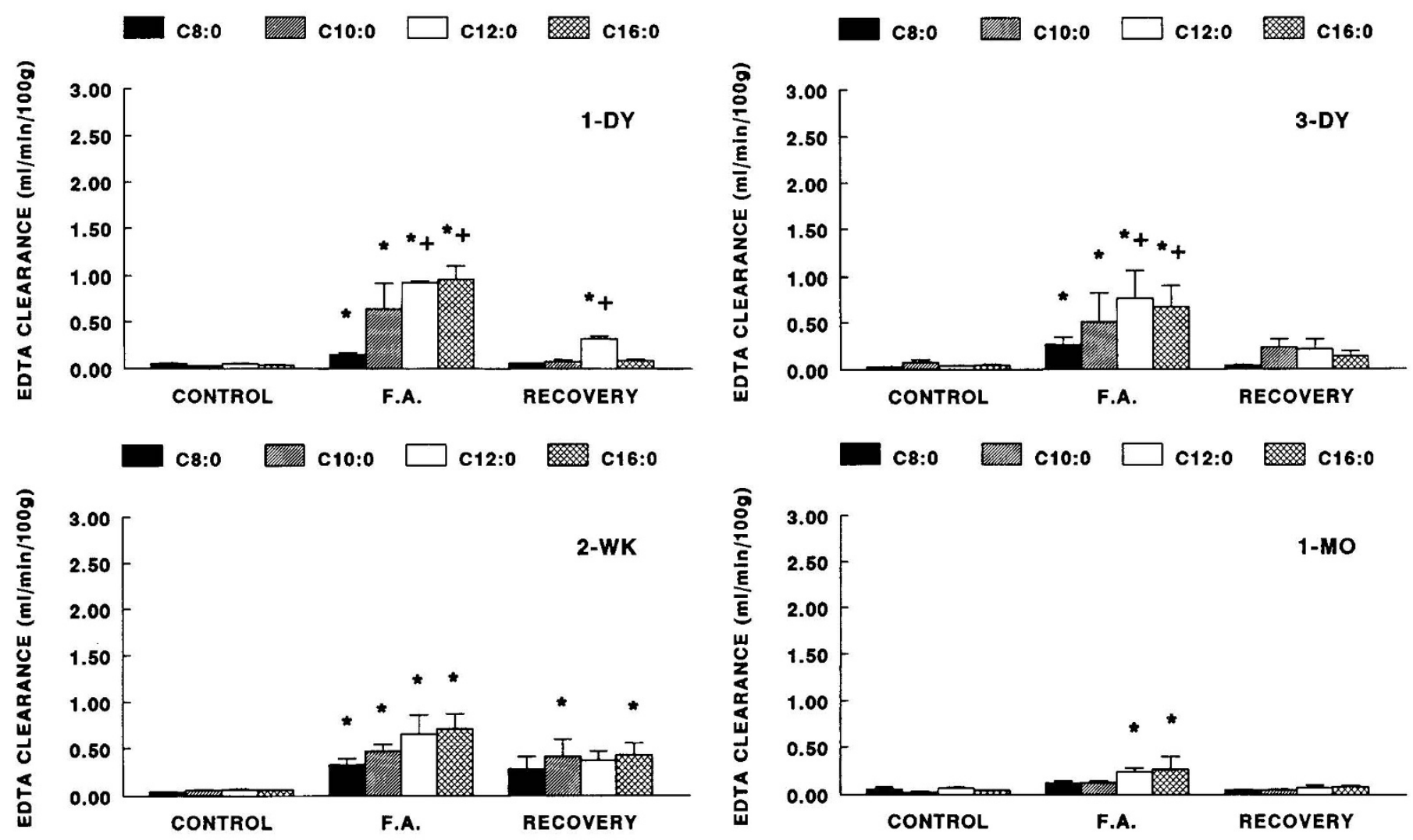

Fig. 4. ${ }^{51} \mathrm{Cr}$-EDTA clearance after perfusion with saturated MCFA and LCFA in jejunum. Values were obtained after control, F.A. (peak increase at time $50 \mathrm{~min}$ ), and recovery periods in intestine of 1-d-, 3-d-, 2-wk-, and 1-mo-old piglets. ${ }^{*}, p<0.05 v s$ control within a fatty acid solution.,$+ p$ $<0.05$ vs caprylic acid (C8:0) within an experimental period.

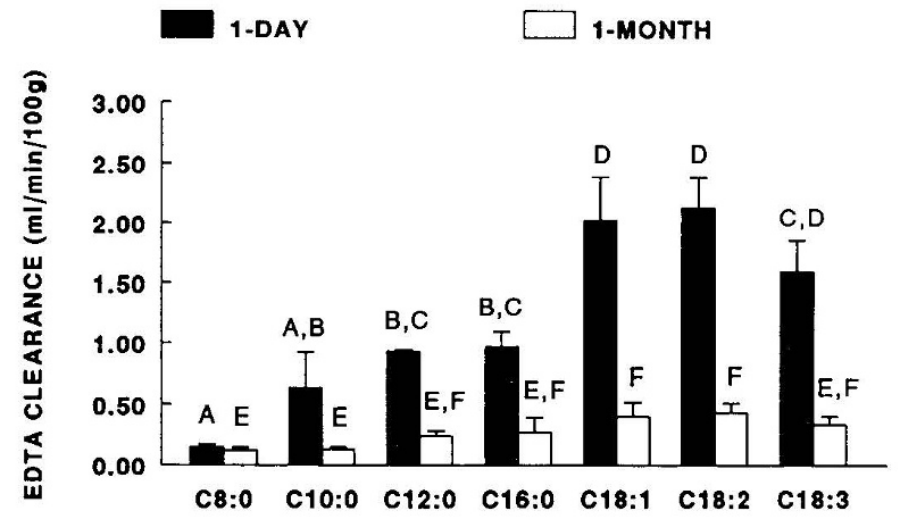

Fig. 5. Effects of MCFA and LCFA on mucosal permeability in developing piglet jejunum. Values represent the peak increase in ${ }^{51} \mathrm{Cr}$ EDTA clearance observed after the F.A. period. Similar letters indicate statistically similar values $(p>0.05)$. With the exception of $\mathrm{C} 8: 0$ and $\mathrm{C} 10: 0$, all permeability values in 1 -d-old piglets were significantly higher $(p<0.05)$ compared with those in 1 -mo-old animals.

\section{DISCUSSION}

Our results demonstrate that perfusion of different MCFA and LCFA, at a concentration of $5 \mathrm{mM}$, which should certainly be present postprandially in the intestinal lumen, produces mucosal injury in developing piglet intestine. The sensitivity of the immature intestine to fatty acid-induced injury decreases with increasing age. This injury is dependent on the carbon chain length of the fatty acid such that severity of the mucosal injury is proportional to the length of the carbon chain. The degree of saturation does not appear to influence the injurious effect of the LCFA. Furthermore, the fatty acid-induced mucosal injury reverses with time. We have shown that mucosal healing at the end of the recovery period is associated with the presence of flattened epithelial cells covering denuded lamina propria, which suggests restitution of the mucosa (7).

Because the injury occurs within minutes after the intestinal mucosa is exposed to fatty acids (Fig. 1), a direct effect of the lipid on the mucosal barrier has been suggested. Fatty acids in solution at $\mathrm{pH} 7.4$ (pKa $\sim 5$ ), behave as amphiphile molecules; that is, they are partly hydrophilic and partly hydrophobic. Therefore, these compounds can act as detergents. Detergents bind to biologic membranes even at very low concentrations, and this binding affects membrane properties in many ways. These changes may involve subtle alterations in permeability or, at higher detergent concentrations, more drastic effects such as membrane lysis and fusion (11). A concentration-dependent injury has also been observed in intestinal mucosa of developing piglets (7) and adult rats (8) after perfusion with bile acidsolubilized oleic acid. In fact, the concentration of fatty acids used in this study $(5 \mathrm{mM})$ has been shown to produce a 20 -fold increase in mucosal permeability in 1-d-old piglet jejunum (7) and is associated with cell injury observed with light and electron microscopy (7). Of interest is that adult rat jejunum is insensitive to the effects of oleic acid in concentrations less than $20 \mathrm{mM}$. In spite of the species difference, this observation indicates that fatty acids are more injurious in newborn animals due to some unknown characteristic of immature intestine.

The carbon chain length-dependent mucosal injury observed in this study supports the hypothesis of a direct detergent action of fatty acids on the intestinal cell barrier. It is well known that hydrophobicity of the carbon chain of fatty acid decreases in proportion to its length. Therefore, the dual hydrophilic and hydrophobic characteristics of a detergent molecule are lost in fatty acids of short chain length. This may lead to a decreased affinity and binding of the amphiphile to the membrane lipid bilayer and also to a decreased ability to form mixed micelles 
and to solubilize membranes, because the hydrophilic groups oppose micelle formation (11).

Previous work from our laboratory has reported that perfusion of piglet intestine with $10 \mathrm{mM}$ sodium taurocholate, the solubilizing agent used in this study, does not alter mucosal permeability to EDTA (7). Bile salts are classified as detergents, and this observation argues against the detergent effect as an explanation for the mucosal injury observed. However, bile salts may still play a synergistic role in the pathophysiology of the lipid-associated injury reported. The precise mechanisms of the fatty acidinduced injury in developing intestine, however, remain to be elucidated.

Because lipid-induced mucosal injury in developing intestine is related to the carbon chain length of the fatty acids, it is tempting to propose this characteristic as an advantage of MCFA over LCFA. This would support the current practice of using fat blends in premature infant formulas containing relatively high levels of MCFA [40-50\% of the total fatty acids (12)]. The rationale for using such high concentrations of MCFA is based on the assumption that: 1) MCFA do not require solubilization with bile salts and, therefore, are absorbed easily by the gastrointestinal tract, including gastric mucosa (13); 2) MCFA are transported directly to the liver via the portal vein, where they are metabolized without the need for carnitine-mediated transport (14); and 3) the rapid oxidation of MCFA and subsequent energy production may improve nitrogen retention and growth of the preterm neonates (12). Despite these important theoretical advantages, feeding the neonate MCFA at concentrations exceeding the infant's requirements may not necessarily be better and in fact may be detrimental (15). Our study showed that lauric acid (C12:0), an MCFA, produced mucosal injury comparable to that observed with LCFA in newborn intestine (Fig. 5). Additionally, MCFA cannot be transformed by the body into essential LCFA, which are used as building blocks in cell membranes. This is a critical metabolic event for the normal maturation of vital organs (e.g. brain) and can only be achieved by the addition of essential LCFA in the diet.

A last interesting observation derived from this study is that the sensitivity to mucosal injury induced by fatty acids is dependent on developmental age. Although we do not have a clear explanation for this phenomenon, other investigators have shown modifications in lipid composition of cell membranes in intestinal mucosa of developing mammals, including piglets (1618). Differences in lipid composition of intestinal apical membranes may have a functional significance, and it has been suggested that these changes lead to a decrease in plasma membrane fluidity and intestinal permeability (19). Whether these observations can provide a rationale for the postnatal differences observed in our study remains to be investigated.

In summary, we have shown that fatty acids, at concentrations present postprandially, induce age- and carbon chain lengthdependent mucosal injury in developing piglet intestine. Although MCFA seem to be overall less injurious than LCFA and play an important role as a source of energy in the preterm neonate, LCFA are essential for growth, development, and maturation of vital organs. Most of these nutrients cannot be synthesized by the body and therefore must be provided in the diet. Finding the means to supply essential LCFA without concomitantly increasing intestinal mucosal injury may provide alternative ways to feed premature infants at risk of gastrointestinal pathology.

\section{REFERENCES}

1. Aggett PJ, Haschke F, Heine W, Hernell O, Koletzko B, Launiala K, Rey J, Rubino A, Schoch G, Senterre J, Tormo R 1991 Comment on the content and composition of lipids in infant formulas. Acta Paediatr Scand 80:887896

2. Finley AJ, Davidson M 1980 Bile acid excretion and patterns of fatty acid absorption in formula-fed premature infants. Pediatrics 65:132-138

3. Lebenthal E, Lee PC 1980 Development of functional response in human exocrine pancreas. Pediatrics 66:556-560

4. Hamosh M, Mehta NR, Fink CS, Coleman J, Hamosh P 1991 Fat absorption in premature infants: medium-chain triglycerides and long-chain triglycerides are absorbed from formula at similar rates. J Pediatr Gastroenterol Nutr 13:143-149

5. Hernell O, Bläckberg L, Bernbäck S 1989 Digestion of human milk fat in early infancy. Acta Paediatr Scand 351:57-62

6. Crissinger KD, Tso $P 1992$ The role of lipids in ischemia/reperfusion-induced mucosal injury in developing piglets. Gastroenterology 102:1693-1699

7. Velasquez OR, Henninger K, Fowler M, Tso P, Crissinger KD 1993 Oleic acid-induced mucosal injury in developing piglet intestine. Am J Physiol 264:G576-G582

8. Kvietys PR, Specian RD, Grisham MB, Tso P 1991 Jejunal mucosal injury and restitution: role of hydrolytic products of food digestion. Am J Physiol 261:G384-G391

9. Gaginella TS, Lewis JC, Phillips SF 1977 Rabbit ileal mucosa exposed to fatty acids, bile acids, and other secretagogues. Dig Dis 22:781-790

10. Iverson SJ, Kirk CL, Hamosh M, Newsome J 1991 Milk lipid digestion in the neonatal dog: the combined actions of gastric and bile salt stimulated lipases. Biochim Biophys Acta 1083:109-119

11. Helenius A, Simons K 1975 Solubilization of membranes by detergents. Biochim Biophys Acta 415:29-79

12. Borum PR 1992 Medium-chain triglycerides in formula for preterm neonates: implications for hepatic and extrahepatic metabolism. J Pediatr 120:S139S145

13. Hamosh M, Bitman J, Liao TH, Mehta NR, Buczek RJ, Wood DL, Grylack LJ, Hamosh P 1989 Gastric lipolysis and fat absorption in preterm infants: effect of MCT or LCT containing formulas. Pediatrics 83:86-92

14. Bach AC, Babayan VK 1982 Medium-chain triglycerides: an update. Am J Clin Nutr 36:950-962

15. Hamosh, M, Spear ML, Bitman J, Mehta NR, Wood DL, Hamosh P 1991 Medium chain triglycerides: advantages and possible drawbacks. In: Schaub $\mathrm{J}$, van Hoof F, Vis HL (eds) Inborn Errors of Metabolism. Raven Press, New York, pp 81-90

16. Alessandri J-M, Guesnet P, Arfi TF, Durand G 1991 Changes in fatty acid composition during cell differentiation in the small intestine of suckling piglets. Biochim Biophys Acta 1086:340-348

17. Chu SW, Walker A 1988 Development of the gastrointestinal mucosal barrier: changes in phospholipid head groups and fatty acid composition of intestinal microvillus membranes from newborn and adult rats. Pediatr Res 23:439442

18. Pang KY, Bresson JL, Walker JL 1983 Development of the gastrointestinal mucosal barrier: evidence for structural differences in microvillus membranes from newborn and adult rabbits. Biochim Biophys Acta 727:201-208

19. Meddings JB, Theisen S 1989 Development of rat jejunum: lipid permeability, physical properties, and chemical composition. Am J Physiol 256:G931G940 